\title{
A importância do ensino de pós-graduação na formação de recursos humanos para o estudo da biodiversidade no Brasil: um estudo de caso na ornitologia
}

\author{
Sérgio Henrique Borges ${ }^{1,2}$ \\ ${ }^{1}$ Fundação Vitória Amazônica, R. Estrela D’Alva, casa 07, Conjunto Morada do Sol, Aleixo, \\ CEP 69060-510, Manaus, AM, Brasil \\ ${ }^{2}$ Autor para correpondência: Sérgio Henrique Borges, e-mail: sergio@fva.org.br
}

Borges, S. H. The relevance of graduate teachings in the formation of human resources for biodiversity studies in Brazil: a case study in ornithology. Biota Neotrop., vol. 8, no. 1, Jan./Mar. 2008. Available from: $<\mathrm{http}: / / w w w . b i o t a n e o t r o p i c a . o r g . b r / v 8 n 1 / e n / a b s t r a c t ?$ point-of-view+bn00108012008>.

\begin{abstract}
Between 1970 and 2005, 397 master's thesis and $108 \mathrm{PhD}$ dissertations with ornithological themes were recorded from 43 Brazilian institutions. Most of these studies were produced in research institutions in Southwestern Brazil. Institutions in the North and Northeastern regions contributed with a relatively low number of ornithological studies. Avifauna was best studied from Coastal and Marine biomes, Atlantic Forest and Cerrado, while research on birds in Caatinga and Amazonia was relatively scarce. Since 1990, were observed a significant increase in scientific production of ornithological studies in graduate courses. The thematic diversity of the courses also increased, but ornithological research in graduate courses is still dominated by community, behavior and synecological studies. Emergent research programs such as molecular systematics, macroecology and landscape ecology have been recently explored in graduate courses in Brazilian institutions. Increasing graduate programs and continuing the thematic diversification of ornithological studies are the challenges that will be faced by Brazilian graduate programs in the next coming years.
\end{abstract}

Keywords: Brazilian ornithology, human resources, master's thesis, PhD dissertations, research programs.

Borges, S. H. A importância do ensino de pós-graduação na formação de recursos humanos para o estudo da biodiversidade no Brasil: um estudo de caso na ornitologia. Biota Neotrop., vol. 8, no. 1, jan./mar. 2008. Disponível em: <http://www.biotaneotropica.org.br/v8n1/pt/abstract?point-of-view+bn00108012008>.

Resumo: Entre 1970 e 2005 foram produzidas 397 dissertações de mestrado e 108 teses de doutorado com temas ornitológicos em 43 instituições brasileiras. A maioria dos estudos foi produzida em instituições localizadas na região sudeste. Instituições do norte e nordeste contribuíram com um número relativamente baixo de estudos ornitológicos. As avifaunas dos biomas Costeiro e Marinho, a Mata Atlântica e o Cerrado foram as mais bem estudadas, enquanto um baixo número de pesquisas foi realizado na Caatinga e na Amazônia. Desde 1990 foi observado um expressivo aumento na produção científica de estudos ornitológicos nos cursos de pós-graduação do Brasil. A diversidade temática dos estudos também se ampliou ao longo dos anos, mas ainda é dominada por estudos de comunidade, comportamento e sinecologia. Temas emergentes como sistemática molecular, macroecologia e ecologia de paisagens já começam a ser explorados nos cursos de pós-graduação. A ampliação dos programas de pós-graduação e a contínua diversificação temática dos estudos ornitológicos são os desafios para os cursos destes programas no Brasil nos próximos anos.

Palavras-chave: dissertações de mestrado, recursos humanos, ornitologia brasileira, temas de pesquisa, teses de doutorado. 


\section{Introdução}

A formação quantitativa e qualitativa de recursos humanos especializados é uma das etapas mais importantes para o amadurecimento de determinada área do conhecimento científico em qualquer país. Neste aspecto, os cursos de pós-graduação desempenham um papel estratégico. A modernização dos cursos de pós-graduação no Brasil iniciou-se no final da década de 1960 e alcançou um notável crescimento nos últimos vinte anos (Beiguelman 1990). Na década de 1990, por exemplo, se contabilizavam mais de 32 mil doutores no Brasil e apenas um em cada cinco doutores foram formados em instituições estrangeiras (Guimarães et al. 2001, Marchelli 2005). Na última década também houve um expressivo aumento de doutores na população brasileira passando de 0,82 para 4,6 doutores por 100 mil habitantes (Marchelli 2005). Apesar destes avanços, ainda persistem desigualdades regionais na formação de mestres e doutores e no repasse de recursos para a consolidação dos cursos de pós-graduação no país (Bortolozzi \& Gremski 2004).

Apesar de existirem análises gerais sobre os impactos da pósgraduação na formação de recursos humanos no Brasil (Beiguelman 1990, Bortolozzi \& Gremski 2004, Marchelli 2005, Steiner 2005), poucos estudos avaliaram a importância destes cursos no desenvolvimento de áreas específicas do conhecimento. Avaliações da contribuição dos cursos de pós-graduação para o avanço destas áreas podem ajudar a conhecer melhor as necessidades de pesquisadores em áreas temáticas relevantes como o estudo da enorme biodiversidade do Brasil (Lewinsohn \& Prado 2002).

Em análises pioneiras, Borges (1995) e Borges \& Uejima (2000) avaliaram a importância dos cursos de pós-graduação na consolidação da ornitologia do Brasil e identificaram um aumento significativo na formação de mestres e doutores especializados em aves a partir da década de 1990. No presente artigo é realizada uma considerável ampliação dos estudos anteriores com o objetivo de descrever e analisar a importância da produção científica dos cursos de pós-graduação para o desenvolvimento recente da ornitologia no Brasil, incluindo avanços quantitativos e tendências temáticas.

\section{Material e Métodos}

Este estudo se baseia na análise de um banco de dados de títulos de dissertações e teses com temas ornitológicos. Para a montagem deste banco de dados foram consultados mais de 80 cursos de pós-graduação nas áreas de zoologia, ecologia, biologia animal, agronomia, engenharia florestal, genética entre outros. O esforço de compilação destes títulos contou com o auxílio de vários orientadores, coordenadores e alunos dos cursos consultados (ver agradecimentos). Também foram consultadas páginas de internet da Universidade Estadual de Campinas (www. libdig.unicamp.br), Universidade Federal de Mato Grosso (www.ufmt. br), Universidade Federal de Minas Gerais (www.icb.ufmg.br) e da Coordenação de Aperfeiçoamento de Pessoal de Nível Superior-CAPES (www.capes.gov.br). Este último recurso foi utilizado de duas maneiras complementares. Inicialmente foram consultadas as relações nominais dos títulos de dissertações e teses dos cursos de pós-graduação do país defendidas desde 1998. Esta busca foi realizada curso a curso dando especial ênfase aos cursos de zoologia, ecologia, engenharia florestal, agronomia e genética. Num segundo momento foi feita uma busca no banco de teses da CAPES (http://servicos.capes.gov.br/capesdw/) que oferece a possibilidade de busca por palavras-chave em cada uma das universidades ou institutos de pesquisa. As palavras-chave utilizadas foram "ornitologia", "avifauna" "e "aves", e optou-se pela busca selecionada por "qualquer uma das palavras". Neste caso a busca foi realizada em 51 universidades ou institutos de pesquisas (Apêndice 1). Utilizando essa estratégia foi possível encontrar estudos sobre aves silvestres no Brasil realizados em cursos de pós-graduação que não foram alvos de busca sistemática (p.ex. multimeios, psicologia experimental). As consultas aos coordenadores de cursos de pós-graduação, a busca em sites de universidades e no site da CAPES permitiram uma compilação bastante extensa dos títulos de teses e dissertações com temas ornitológicos elaboradas em entidades brasileiras.

Para se analisar a diversidade temática dos estudos ornitológicos, os títulos de dissertações e teses foram categorizados em temas de estudo. Inicialmente foi feita uma categorização geral utilizando os seguintes temas: biogeografia, comportamento, comunidade, conservação, doenças em aves, educação ambiental, etnozoologia, faunística, fisiologia, genética, histologia, história da ciência, manejo, mercado, metodologia, microbiologia, morfologia, parasitismo, sinecologia (estudo de espécies individuais), sistemática e taxonomia. Para uma análise temática mais refinada, os títulos de dissertações e teses foram ainda categorizados em 53 temas mais específicos (p. ex. anatomia comparada, ecologia de paisagens, fragmentação). Em alguns casos os títulos das dissertações e teses não permitiram definir claramente os temas de estudo e por isso esta categorização mais específica nem sempre foi possível. Algumas vezes a categorização das teses e dissertações em temas gerais e específicos envolveu algumas escolhas arbitrárias. Apesar disto, este exercício permitiu a identificação de tendências nos estudos ornitológicos no país.

A diversidade temática das dissertações e teses foi analisada através do índice de diversidade Shannon-Wienner (Magurran 1998) calculado para cada ano no qual foi produzida mais de uma dissertação ou tese. A correlação entre a diversidade temática e os anos foi testada através da análise de correlação de Spearman.

\section{Resultados}

\section{Tendências gerais}

Foi compilada uma listagem de 397 títulos de dissertações de mestrado e 108 de teses de doutorado que tratam de estudos sobre a biologia de aves em 43 instituições de ensino e pesquisa no Brasil (uma listagem completa dos títulos, incluindo a categorização temática, pode ser solicitada ao autor). A produção destes estudos apresentou uma grande variabilidade inter-anual (Figura 1). Houve um aumento consistente na produção de estudos ornitológicos produzidos nos cursos de pós-graduação a partir de meados da década de 1990 no nível de mestrado e a partir de 2000 no de doutorado (Figura 1).

Grande parte dos estudos analisados $(57 \%)$ foi produzida em instituições localizadas na região sudeste, onde se concentra a maioria dos cursos de pós-graduação do país (Tabela 1). Uma boa proporção de estudos também foi produzida em instituições do sul (18\%) e centrooeste (12\%). Em contraste, instituições das regiões norte e nordeste contribuíram com um número relativamente baixo de estudos ornitológicos. A Universidade de São Paulo (USP), principalmente nos cursos de genética, ecologia e zoologia, foi responsável pelo maior número de dissertações e teses - 13\% do total (Tabela 1). A Universidade de Brasília (UnB) (cursos de ecologia e biologia animal) também se destacou produzindo $9 \%$ das dissertações e teses com temas ornitológicos.

Relativizando a diversidade de espécies de aves nos biomas brasileiros (Marini \& Garcia 2005) pelo número de estudos de pósgraduação, os biomas mais bem estudados são o Costeiro e Marinho (um estudo para cada três espécies, $\mathrm{N}=47$ estudos), a Mata Atlântica (um estudo para cada 8 espécies, $\mathrm{N}=123$ ) e o Cerrado (um estudo para cada 16 espécies, $\mathrm{N}=52$ ). A Caatinga (um estudo para cada 102 espécies, $\mathrm{N}=5$ ) e a Amazônia (um estudo para cada 38 espécies, $\mathrm{N}=34$ ) são os biomas com menor número relativo de estudos. Os biomas Campos Sulinos (um estudo para cada 23 espécies, $\mathrm{N}=21$ ) e Pantanal (um estudo para cada 33 espécies, $\mathrm{N}=14$ ) ocupam posições intermediárias na produção de estudos de pós-graduação.

Contabilizando somente as espécies indicadas nos títulos das dissertações e teses, somente 137 (8\%) das aproximadamente 1.700 espécies 


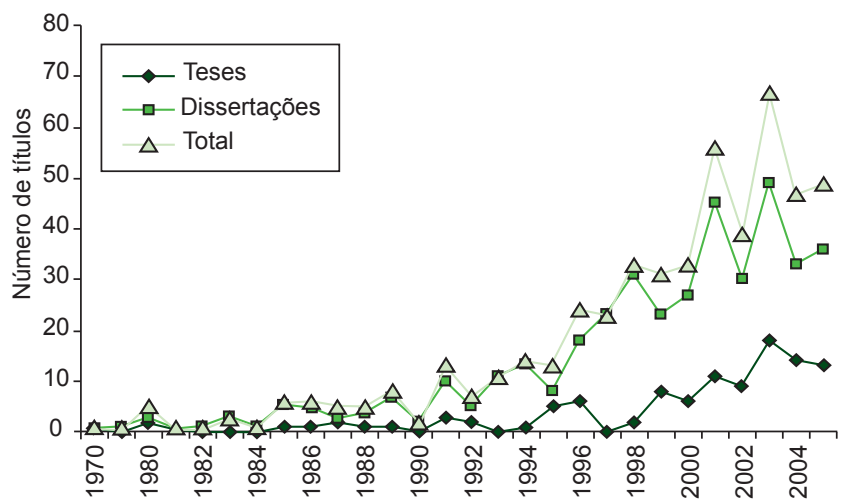

Figura 1. Produção quantitativa de dissertações de mestrado e teses de doutorado versando sobre a biologia de aves produzidas em instituições de ensino e pesquisa no Brasil.

Figure 1. Quantitative production of master's thesis $(\mathrm{M})$ and $\mathrm{PhD}$ dissertations (D) with bird biology thematics made in graduate courses in the Brazilian institutions).

de aves brasileiras foram alvos de estudos específicos. Estes estudos incluem desde espécies muito comuns como o anu-branco Guira guira e o tiziu Volatinia jacarina (Almeida 1997, Melo 1997) até espécies mais raras e recentemente descritas como bicudinho-do-brejo Stymphalornis acutirostris e o soldadinho-do-araripe Antilophia bokermanni (Reinert 2001, Silva 2004). A biologia de somente 11 das 160 espécies que constam da listagem de aves ameaçadas de extinção reconhecidas pelo Ibama (Ibama 2003) foi analisada em estudos de pós-graduação (p. ex. Neto 1989, Pauletti 1996, Mendonça 2001).

\section{Diversidade temática}

A diversidade temática no estudo das aves nos cursos de pósgraduação aumentou substancialmente ao longo dos anos (Figura 2), existindo uma forte correlação entre os anos e a diversidade de temas gerais ( $\mathrm{rs}=0.68, \mathrm{P}<0.001)$ e específicos ( $\mathrm{rs}=0.88, \mathrm{P}<0.001)$.

Como identificado nos estudos anteriores (Borges 1995, Borges \& Uejima 2000), as pesquisas com aves ainda são dominadas por estudos de comunidade, sinecologia e comportamento (Figura 3). Sinecologia e faunística são temas mais frequentes nos cursos de mestrado, enquanto estudos de sistemática e taxonomia e genética são mais comuns nos cursos de doutorado (Figura 3).

Os temas de estudos específicos mais frequentes são biologia reprodutiva ( $9 \%$ dos títulos), fragmentação ( $8 \%$ dos títulos), conservação (8\% dos títulos), ecologia alimentar ( $8 \%$ dos títulos) e frugivoria (5\% dos títulos). Temas emergentes como macroecologia, ecologia de paisagens e sistemática molecular e morfológica ainda são escassos, mas já começam a ser trabalhados nos cursos de pós-graduação (p. ex. Zimmer 1992, Raposo 1996, Miyaki 1996, Sant'ana 1998, Dário 1999, Gonzaga 2001).

\section{Discussão}

\section{A importância da pós-graduação para a ornitologia no Brasil}

A história da ornitologia no Brasil foi dividida por Alves \& Silva (2000) nos períodos das explorações estrangeiras (1500 ao século XIX), dos museus de história natural (meados do século XIX até a década de 1970) e no período moderno a partir dos anos 1970 no qual se destacam as pesquisas realizadas nas universidades. O período moderno da ornitologia no Brasil se beneficiou amplamente dos cursos de pós-graduação que começaram a se reestruturar e receber mais apoio do governo brasileiro a partir da década de 1970 (Beiguelman
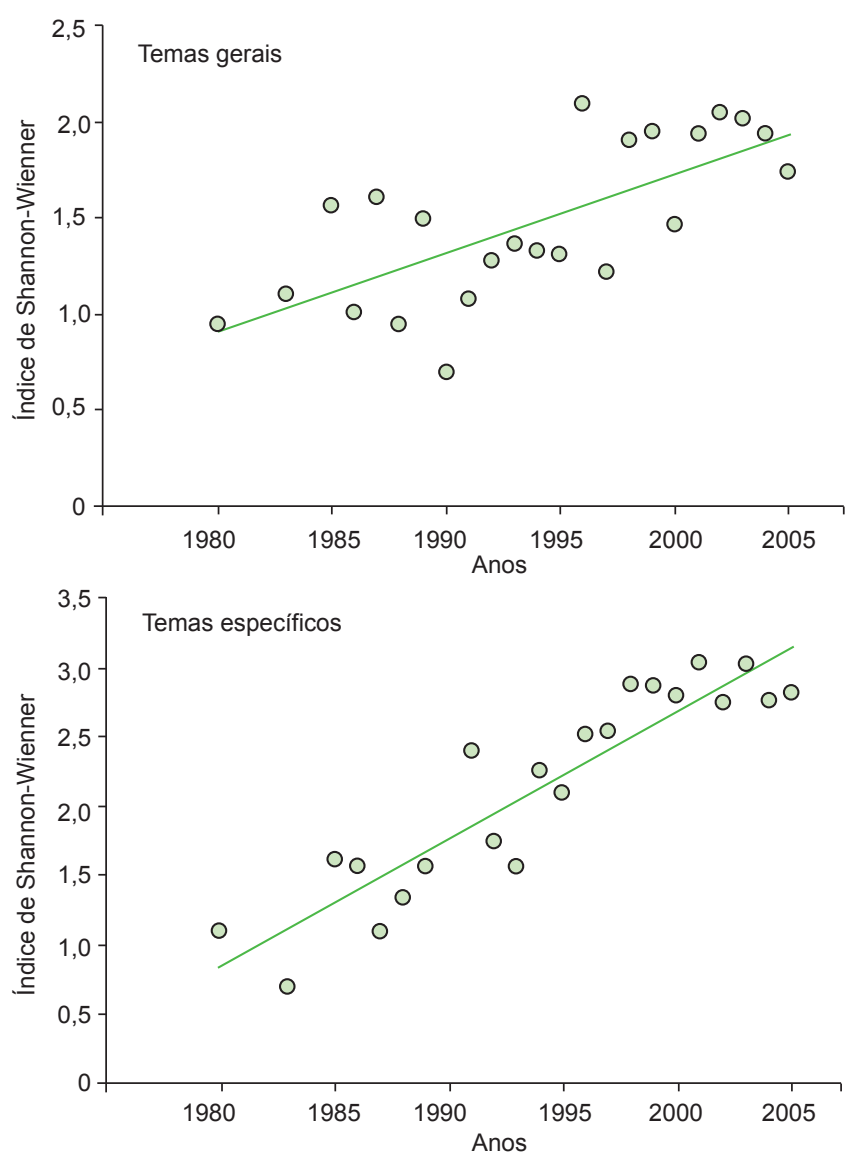

Figura 2. Evolução temporal da diversidade temática (medida pelo índice de Shannon-Wienner) dos estudos ornitológicos realizados nos cursos de pós-graduação no Brasil.

Figure 2. Temporal evolution of general (above) and specific (below) thematic diversity of ornithological studies measured by the Shannon-Wienner diversity index.

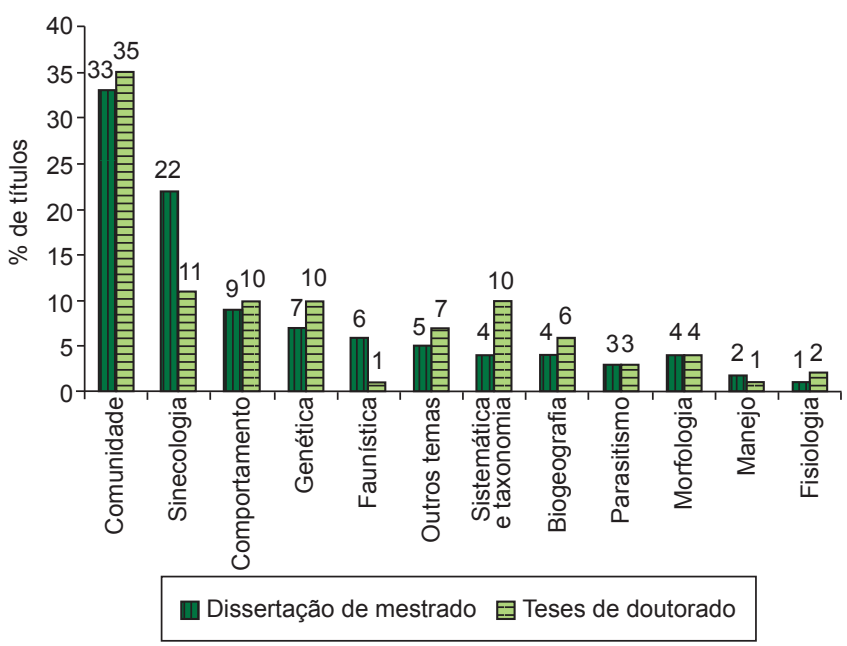

Figura 3. Temas gerais de estudos ornitológicos explorados nos cursos de pós-graduação no Brasil. Outros temas são aqueles com menos de seis títulos e incluem doenças em aves, conservação, história da ciência, histologia, metodologia, etnozoologia, educação ambiental, mercado e microbiologia

Figure 3. General themes of ornithological studies explored in graduate courses in Brazil. Other themes are those with less than six titles in the database and include bird health, conservation, history of science, histology, methodology, ethnozoology, environmental education, market and microbiology. 
Borges, S. H

Tabela 1. Número de dissertações de mestrado $(\mathrm{M})$ e teses de doutorado (D) que tratam de temas relacionados à biologia de aves elaboradas em cursos de pós-graduação no Brasil no período de 1970 a 2005. As teses de livre-docência foram computadas junto com as de doutorado.

Table 1. Number of master's dissertations (M) and PhD theses (D) with bird biology themes made in graduate courses in the Brazilian institutions from 1970 to 2005 .

\begin{tabular}{|c|c|c|c|c|c|c|c|c|c|}
\hline \multirow[t]{2}{*}{ Instituiçõos } & \multicolumn{2}{|c|}{ Ecologia } & \multicolumn{2}{|c|}{ Genética } & \multicolumn{2}{|c|}{ Outros Cursos* } & \multicolumn{2}{|c|}{ Zoologia** } & \multirow[t]{2}{*}{ Total geral } \\
\hline & D & $\mathbf{M}$ & D & M & D & M & D & M & \\
\hline Sudeste total & 21 & 70 & 10 & 24 & 8 & 50 & 35 & 71 & 289 \\
\hline USP & 2 & 10 & 8 & 7 & 2 & 14 & 14 & 9 & 66 \\
\hline UNESP/RC & - & - & - & - & - & 3 & 14 & 19 & 36 \\
\hline UFMG & 3 & 21 & - & 2 & 1 & 5 & - & - & 32 \\
\hline UNICAMP & 7 & 20 & - & - & 1 & 3 & - & - & 31 \\
\hline UFSCAR & 9 & 9 & 1 & 6 & - & - & - & - & 25 \\
\hline UFRJ & - & 2 & - & 1 & - & - & 2 & 18 & 23 \\
\hline UFRRJ & - & - & - & - & - & 4 & 4 & 9 & 17 \\
\hline UNESP/Bot. & - & - & 1 & 7 & - & 1 & 1 & 7 & 17 \\
\hline USP/Esalq & - & 3 & - & - & - & 7 & - & - & 10 \\
\hline $\mathrm{PUC} / \mathrm{MG}$ & - & - & - & - & - & - & - & 8 & 8 \\
\hline UERJ & - & - & - & - & 2 & 3 & - & - & 5 \\
\hline UFU & - & 5 & - & - & - & - & - & - & 5 \\
\hline UFLA & - & - & - & - & - & 3 & - & - & 3 \\
\hline USP/SÃO CARLOS & - & - & - & - & 1 & 2 & - & - & 3 \\
\hline FIOCRUZ & - & - & - & - & 1 & 1 & - & - & 2 \\
\hline UFF & - & - & - & - & - & 2 & - & - & 2 \\
\hline UFV & - & - & - & - & - & 2 & - & - & 2 \\
\hline UNESP/SJRP & - & - & - & - & - & - & - & 1 & 1 \\
\hline USP/RP & - & - & - & 1 & - & - & - & - & 1 \\
\hline Sul total & 1 & 16 & 1 & 4 & - & 21 & 10 & 36 & 89 \\
\hline UFPR & - & - & - & - & - & 7 & 6 & 16 & 29 \\
\hline PUC/RS & - & - & - & - & - & - & 4 & 18 & 22 \\
\hline UFRGS & - & 10 & 1 & 4 & - & - & - & 2 & 17 \\
\hline FURGS & - & - & - & - & - & 5 & - & - & 5 \\
\hline UEL & - & 2 & - & - & - & 2 & - & - & 4 \\
\hline UEM & 1 & 3 & - & - & - & - & - & - & 4 \\
\hline UNIVALI & - & - & - & - & - & 3 & - & - & 3 \\
\hline UFSC & - & - & - & - & - & 4 & - & - & 4 \\
\hline UNISINOS & - & 1 & - & - & - & - & - & - & 1 \\
\hline Centro-oeste total & 7 & 42 & - & - & - & 6 & 4 & 3 & 62 \\
\hline UnB & 7 & 28 & - & - & - & 1 & 4 & 3 & 43 \\
\hline UFMS & - & 9 & - & - & - & - & - & - & 9 \\
\hline UFMT & - & 5 & - & - & - & - & - & - & 5 \\
\hline UFG & - & - & - & - & - & 4 & - & - & 4 \\
\hline UNIDERP & - & - & - & - & - & 1 & - & - & 1 \\
\hline Norte total & 1 & 15 & 1 & 2 & 4 & 3 & 3 & 8 & 37 \\
\hline UFPA & - & - & 1 & 1 & 4 & 2 & 3 & 8 & 19 \\
\hline INPA & 1 & 15 & - & 1 & - & 1 & - & - & 18 \\
\hline Nordeste total & - & 2 & - & - & - & 3 & 6 & 17 & 28 \\
\hline UFPE & - & - & - & - & - & 2 & - & 10 & 12 \\
\hline UFPB & - & - & - & - & - & - & 2 & 7 & 9 \\
\hline UFRPE & - & - & - & - & - & - & 3 & - & 3 \\
\hline UESC & - & - & - & - & - & 1 & - & - & 1 \\
\hline UEFS & - & - & - & - & - & - & 1 & - & 1 \\
\hline UFBA & - & 1 & - & - & - & - & - & - & 1 \\
\hline UFRN & - & 1 & - & - & - & - & - & - & 1 \\
\hline Total geral & 30 & 145 & 12 & 30 & 12 & 83 & 58 & 135 & 505 \\
\hline
\end{tabular}

*Engenharia florestal, Biologia parasitária, Oceanografia biológica, Ciências biológicas (Biociências nucleares - área de concentração em Ecologia), Desenvolvimento regional e meio ambiente, Medicina veterinária, Ciências biológicas (Microbiologia), Morfologia, Parasitologia, Neurociências e biologia celular, Gestão e políticas ambientais, Ciências biológicas (Entomologia), Gestão e estratégia em negócios, Transportes, Ciências biológicas (Biologia vegetal), Multimeios, Meio ambiente e desenvolvimento regional, Ciência e tecnologia ambiental, Turismo e hotelaria, Ciências (Fisiologia geral), Ciências biológicas (Fisiologia), Ciências biológicas (Microbiologia), Patologia experimental e comparada, Psicologia (Neurociências e comportamento), Psicologia (Psicologia experimental), Zootecnia, Ciências da computação e matemática computacional, Ciências da engenharia ambiental. ** Os cursos de zoologia incluem os de biologia animal.

1990). Não é coincidência, portanto, que a primeira tese de doutorado sobre aves produzida numa entidade nacional tenha sido defendida em 1970 (Novaes 1970).

As primeiras décadas dos cursos de pós-graduação, no entanto, se caracterizaram por uma produção pequena e errática de pesquisas sobre aves devido, provavelmente, à escassez de recursos humanos aptos a orientar estes estudos. Neste período muitos ornitólogos buscaram por treinamento em instituições estrangeiras como o fazem até hoje. Entretanto, já existe um bom número de especialistas em aves total ou parcialmente treinados em instituições brasileiras, inclusive orientando em cursos de pós-graduação (p. ex. Dr. Reginaldo Donatelli da UNESP/Botucatu, Dr. Marcos Raposo do Museu 
Nacional, Dr. Alexandre Aleixo do Museu Paraense Emílio Goeldi e Dr. Miguel Marini da UnB, entre outros).

O recente avanço quantitativo nos estudos ornitológicos se deve provavelmente ao aumento recente de especialistas em aves contratados para trabalhar em várias instituições de ensino e pesquisa no Brasil. Outro aspecto importante, é o aumento de cursos disponíveis nas universidades e institutos de pesquisa. Vários cursos recentemente reconhecidos pela CAPES já possuem uma produção, ainda que modesta, de pesquisas ornitológicas (p. ex. Universidade Federal da Bahia, Universidade Federal de Uberlândia). Assim, o aumento da disponibilidade de orientadores e a ampliação dos cursos de pós-graduação podem explicar o recente avanço dos estudos ornitológicos no Brasil.

O desenvolvimento da ornitologia dos países neotropicais tem sido analisado através da produção científica consolidada em artigos científicos publicados (James 1987, Paynter 1991, Winker 1998). Em todas estas análises o Brasil se destaca como um país latino-americano com respeitada produção científica no estudo de aves, junto com Argentina e México. A produção científica medida através de artigos e através de dissertações e teses deve ter uma relação bastante direta, já que vários destes títulos foram e continuam sendo publicados em revistas científicas. Desde modo, os avanços quantitativo e qualitativo dos estudos sobre biologia de aves produzidos nos cursos de pósgraduação indicam que o Brasil continuará a ter um lugar de destaque na ornitologia neotropical.

\section{Perpectivas da ornitologia nos cursos de pós-graduação}

A produção de estudos da biologia de aves em cursos de pósgraduação ampliou-se muito nas últimas décadas. A diversidade de temas explorados nestes estudos também aumentou bastante, tornando a ornitologia uma ciência ainda mais multidisciplinar. Apesar deste cenário otimista, é importante questionar se os recursos humanos especializados em ornitologia são suficientes para estudar uma avifauna tão diversificada quanto a do Brasil. O Brasil possuí uma das avifaunas mais ricas do mundo com um número de espécies estimado em quase 1.700 , o que equivale a $57 \%$ das espécies de aves registradas na América do Sul (Marini \& Garcia 2005). A maior floresta tropical do planeta, a Amazônia, tem a maior parte de seu território no Brasil, onde ocorrem, ainda, biomas únicos como a Caatinga e o Pantanal. A Mata Atlântica é o bioma que sofreu o mais alto grau de degração ambiental no país e possuí um elevado número de espécies de aves endêmicas e ameaçadas de extinção (Marini \& Garcia 2005). Os desafios de estudar a avifauna brasileira, portanto, não são pequenos e algumas tendências observadas no presente estudo podem ajudar na definição de algumas estratégias de trabalho.

Os resultados do presente estudo indicam que a maior parte das espécies de aves brasileiras ainda não foi alvo de estudos específicos de pós-graduação. A taxa de estudos ornitológicos publicados de 1979 a 1995 em relação à diversidade de aves nos Estados Unidos, um dos países com a avifauna mais bem estudada do mundo, foi de 5,361 enquanto que no Brasil esta mesma taxa foi cerca de dez vezes menor -0,514 (Winker 1998). Deste modo, o estudo dos vários aspectos da biologia ou ecologia de espécies de aves, especialmente aquelas de interesse para a conservação, continua sendo um dos temas mais relevantes a ser incrementado nos cursos de pós-graduação.

Devem ser incentivados estudos com aves da Caatinga e Amazônia, biomas únicos e ricos em espécies, mas com avifaunas ainda pouco estudadas nos cursos de pós-graduação do país. Neste sentido é importante ampliar o suporte aos poucos cursos existentes no norte e nordeste. Para tanto é necessário ampliar o quadro de pesquisadores especializados em aves nos cursos já existentes e alguns recentemente criados nestas regiões (p. ex. cursos de ecologia da Universidade Federal do Acre e da Universidade Federal da Bahia). Os cursos de pós-graduação das entidades do centro-oeste (UnB, UFMT e UFMS) devem dar maior atenção para a avifauna do Pantanal que tem sido relativamente pouco estudada.

Os estudos de biogeografia, taxonomia e sistemática (molecular, morfológica ou comportamental) são críticos para se definir os limites das unidades evolutivas (espécies) e as relações históricas entre regiões tendo importantes implicações para a conservação de aves (Raposo 2001). Estes estudos são, ainda, pouco frequentes nas instituições de ensino e pesquisa no Brasil. Por exemplo, a maior parte dos poucos estudos em sistemática molecular de aves neste país foi realizada no curso de genética da Universidade de São Paulo. Felizmente, existe uma tendência ao aumento destes estudos nas instituições brasileiras. Cursos de genética recentemente reconhecidos no norte do Brasil (INPA e UFPA) deverão ampliar estes estudos para regiões de grande diversidade de aves. A interação produtiva, que já vem acontecendo e tende a se ampliar, entre pesquisadores dos museus de história natural e dos departamentos de zoologia e genética das universidades, certamente resultarão em um aumento substancial de estudos de biogeografia, taxonomia e sistemática de aves no Brasil.

Estudos de fragmentação são relativamente freqüentes nas instituições de pesquisa brasileiras. Estes estudos têm ajudado a entender melhor os efeitos de fragmentação na biota de vários ecossistemas, especialmente da Mata Atlântica e Cerrado (MMA 2003). Os estudos de fragmentação tem mudado de uma perspectiva de entender os fragmentos de ambientes como "ilhas" isoladas para uma abordagem de ecologia de paisagens que incluí um entendimento mais completo da interação entre a biologia de aves e da disposição e característica estruturais dos fragmentos (Wiens 1995). A adoção desta perspectiva de ecologia de paisagens nos estudos sobre aves resultará em pesquisas com alto potencial de aplicação para conservar e proteger as aves em ecossistemas perturbados do Brasil.

A ornitologia no Brasil está passando por uma fase de aumento considerável da produção com um grande número de pesquisas em temas tradicionais (p. ex. comunidades e sinecologia) e emergentes (p. ex. macroecologia e sistemática molecular) sendo realizadas nas universidades e institutos de pesquisa. $\mathrm{O}$ ensino de pós-graduação tem dado uma importante contribuição neste processo. Os desafios a serem enfrentados nos próximos anos são a ampliação dos programas de pós-graduação para regiões e biomas pouco estudados e a contínua diversificação temática, garantindo que a ornitologia no Brasil se consolide como uma ciência moderna, multidisciplinar e alinhada com os padrões internacionais de pesquisa.

\section{Agradecimentos}

A compilação das informações que subsidiaram este estudo só foi possível graças à ajuda e gentileza de uma extensa rede de colaboradores incluindo colegas ornitólogos, coordenadores, secretárias e alunos dos cursos de pós-graduação. Agradeço a disponibilidade e atenção das seguintes pessoas: Josiano Cordeiro Torezani, Márcio Efe, Tutilla de Brito Aragão (Capes), Fernando Pacheco (CBRO), Rudi Laps (FURB), Hercília (INPA), Alexandre (INPA), Marcela de Fátima Torres (INPA), Dorotéa (MPEG), Magali Henriques (MPEG), Ana Harada (MPEG), Arthur Ângelo Bispo (Mülleriana: Sociedade Fritz Müller de Ciências Naturais), Fernando Straube (Mülleriana: Sociedade Fritz Müller de Ciências Naturais), Carlos Rodrigo (Museu Nacional), Nilo Bazzoli (PUC/MG), Carla Fontana (PUC/RS), Nelson Fontoura (PUC/RS), Peixoto (UCG), Luís dos Anjos (UEL), Martín R. Alvarez (UESC), Erminda da Conceição Guerreiro Couto (UESC), Lisandro Juno Soares Vieira (UFAC), Rogério Pereira Bastos (UFG), José Carlos Loures de Oliveira (UFJF), Julio Louzada (UFLA), Mary das Graças Santos (UFMG), Fabrício R. Santos (UFMG), Rogério 
Parentoni (UFMG), Francisco Barbosa (UFMS), Celso Feitosa Martins (UFPB), Adelmar Gomes Bandeira (UFPB), Marcos Morais (UFPE), Vera Maria (UFPR), Paulo Martins (UFRA), Claiton Ferreira (UFRGS), Maria Tereza Raya Rodriques (UFRGS), Francisco MalletRodriques (UFRJ), Sérgio Alex de Azevedo (UFRJ), Marcos Rogerio Camara (UFRN), Roberto Cavalcanti (UnB), Miguel Marini (UnB), Regina Macedo (UnB), Carlos Klink (UnB), Sônia Ciccione (UNESP/ Botucatu), Carlos Henrique Penteado (UNESP/RC), Yoshika Oniki (UNESP/RC), Célia (UNICAMP), Ana Maria Leal Zanchet (UNISINOS), Robson F. Lopes (USP - Esalq), Catarina Germuts (USP Esalq), Felipe Zilio (USP), Jean Paul Metger (USP), Helder Rossi S. Souza (USP), Cristina Miyaki (USP), Astrid de M. P. Kleinert (USP), Astrid de Matos Peixoto (USP). As críticas e sugestões feitas por dois revisores anônimos, em especial a sugestão de estratégias de busca no site da CAPES, enriqueceram sobremaneira este estudo.

Dedicatória: Este estudo é dedicado a todos os orientadores dos cursos de pós-graduação que estão dando uma contribuição inestimável para a consolidação e amadurecimento da ornitologia no Brasil.

\section{Referências bibliográficas}

ALMEIDA, J.B. 1997. Determinantes ecológicos da reprodução de Volatinia jacarina (Passeres, Emberizidae). Dissertação de mestrado, Universidade de Brasília, Brasília.

ALVES, M.A.S. \& SILVA, J.M.C. 2000. A ornitologia no Brasil: desenvolvimento, tendência atuais e perspectivas. In A ornitologia no Brasil: pesquisa atual e perspectivas (M.A.S. Alves, J.M.C. Silva, M.V. Sluys, H.G. Bergallo \& C.F.D da Rocha, orgs.). Rio de Janeiro, Eduerj, p. 327-344.

BANCO DE TESES DA CAPES (http://servicos.capes.gov.br/capesdw/).

BEIGUELMAN, B. 1990. Uma análise crítica da pós-graudação no Brasil. Ciência Hoje, Rio de Janeiro, 12 (68):18-21.

BORGES, S.H. 1995. As teses de pós-graduação produzidas no Brasil na área de ornitologia entre 1970 e 1991. Ararajuba, 3:33-36.

BORGES, S.H. \& UEJIMA, A.M.K. 2000. A importância dos cursos de pósgraduação na formação de ornitólogos no Brasil. In Ornitologia brasileira no século XX - incluindo os Resumos do VIII Congresso Brasileiro de Ornitologia (F.C. Straube, M.M. Argel-de-Oliveira \& J.F. Cândido-Jr, eds.). Universidade do Sul de Santa Catarina e Sociedade Brasileira de Ornitologia, Curitiba, p. 71-75.

BORTOLOZZI, F. \& GREMSKI, W. 2004. Pesquisa e pós-graduação brasileira - assimetrias. Revista Brasileira de Pós-graduação, 1:35-52.

COORDENAÇÃO DE APERFEIÇOAMENTO DE PESSOAL DE NÍVEL SUPERIOR (www.capes.gov.br).

DÁRIO, F.R. 1999. Influência de corredor florestal entre fragmentos da Mata Atlântica utilizando-se a avifauna como indicador ecológico. Dissertação de mestrado, Universidade de São Paulo, Piracicaba.

GONZAGA, L.A.P. 2001. Análise filogenética do gênero Formicivora Swainson, 18 (Aves: Passeriformes: Thamnophilidae) baseada em caracteres morfológicos e vocais. Tese de doutorado, Universidade Federal do Rio de Janeiro, Rio de Janeiro.

GUIMARÃES, R., LOURENÇO, R. \& COSAC, S. 2001. O perfil dos doutores ativos em pesquisa no Brasil. Parcerias Estratégicas, 13:122-150.

IBAMA (INSTITUTO BRASILEIRO DO MEIO AMBIENTE E DOS RECURSOS NATURAIS RENOVÁVEIS). 2003. Lista das espécies da fauna ameaçadas de extinção. Instrução normativa no 3 de 27 de maio de 2003. Ibama, Ministério do Meio Ambiente, Brasília.

JAMES, P.C. 1987. Ornithology in Central and South America. Auk, 104:348-349.

LEWINSOHN, T.M. \& PRADO, P.I. 2002. Biodiversidade Brasileira: síntese do estado atual do conhecimento. Editora Contexto, São Paulo, 176p.

MAGURRAN, A.E. 1988. Ecological diversity and its measurements. Princeton University Press, Princeton, 179p.
MARCHELLI, P.S. 2005. Formação de doutores no Brasil e no mundo: algumas comparações. Revista Brasileira de Pós-graduação, 2:7-29.

MARINI, M.A. \& GARCIA, F.I. 2005. Conservação de aves no Brasil. Megadiversidade, 1:95-102.

MELO, C. 1997. Reprodução comunitária em Guira guira Gmelin 1788 (Cuculidae, Aves): Aspectos eto-ecolóticos. Dissertação de mestrado, Universidade de Brasília, Brasília.

MENDONÇA, E.C. 2001. Biologia Reprodutiva de Formicivora erythronotos (Aves, Thamnophilidae). Dissertação de mestrado, Universidade Federal do Rio de Janeiro, Rio de Janeiro.

MIYAKI, C.Y. 1996. Um estudo filogenético de psitacídeos (Psittaciformes, Aves) baseado em sequências de genes mitocondriais. Tese de doutorado, Universidade de São Paulo, São Paulo.

MMA (MINISTÉRIO DO MEIO AMBIENTE). 2003. Fragmentação de ecossistemas: causas, efeitos sobre a biodiversidade e recomendações de políticas públicas. Secretaria de Biodiversidade e Florestas (SBF), Ministério do Meio Ambiente, Brasília.

NETO, P.S. 1989. Contribuição à biologia do papagaio-da-cara-roxa Amazona brasiliensis L., 1758 (Psittacidae, Aves). Dissertação de mestrado, Universidade Federal do Paraná, Curitiba.

NOVAES, F.C. 1970. Estudo ecológico das aves em uma área de vegetação secundária no Baixo Amazonas, Estado do Pará. Tese de doutorado, Universidade Estadual de São Paulo, Rio Claro.

PAULETTI, N.P. 1996. Estudo eco-etológico de Amazona pretrei Temminck, 1830 (Aves, Psittaciformes), em condição de cativeiro. Dissertação de mestrado, Pontifícia Universidade Católica do Rio Grande do Sul, Porto Alegre.

PAYNTER, R. 1991. The maturation of Brazilian ornithology. Ararajuba, 2:105-106.

RAPOSO, M. 1996. Taxonomia do complexo Hylophilus poicilotis (Aves: Vireonidae). Dissertação de mestrado, Universidade Federal do Rio de Janeiro, Rio de Janeiro.

RAPOSO, M. 2001. Taxonomia alfa de aves neotropicais. In Ornitologia e conservação - da ciência às estratégias (J.L. Albuquerque, J.F. Cândido jr., F.C. Straube, A.L. Roos, eds.). Tubarão, Editora Unisul, p. 249-259.

REINERT, B. L. 2001. Distribuição geográfica, caracterização dos ambientes de ocorrência e conservação do Bicudinho-do-brejo (Stymphalornis acutirostris Bornschein, Reinert \& Teixeira,1995 - Aves, Formicariidae). Dissertação de mestrado, Universidade Federal do Paraná, Curitiba.

SANT'ANA, C.E.R. 1998. Macroecologia e evolução do tamanho do corpo em corujas (Aves: Strigiformes) da América do Sul. Dissertação de mestrado, Universidade Federal de Goiás, Goiânia.

SILVA, W.A.G. 2004. Comportamento de Antilophia bokermanni Coelho \& Silva 1998 (Aves: Pipridae) na Chapada do Araripe - Ceará. Dissertação de mestrado, Universidade Federal de Pernambuco, Recife.

STEINER, J.E. 2005. Qualidade e diversidade institucional na pós-graduação brasileira. Estudos Avançados, 19:341-365.

UNIVERSIDADE ESTADUAL DE CAMPINAS (www.libdig.unicamp.br). UNIVERSIDADE FEDERAL DO MATO GROSSO (www.ufmt.br).

UNIVERSIDADE FEDERAL MINAS GERAIS (www.icb.ufmg.br).

WIENS, J.A. 1995. Habitat fragmentation: island vs. landscape perspective on bird conservation. Ibis, 137:97-104.

WINKER, K. 1998. Recent geographic trends in neotropical avian research. Condor, 100:764-768.

ZIMMER, R. 1992. Análise filogenética de aves da família Anatidae pelo sequenciamento do gene de RNA ribossomal 12s do DNA mitocondrial. Dissertação de mestrado, Universidade Federal do Rio Grande do Sul, Porto Alegre. 


\section{Apêndice 1}

Universidade e institutos de pesquisa brasileiros onde foram feitas buscas para compor o banco de dados de títulos de dissertações e teses com temas ornitológicos.

\section{Appendix 1}

Universities and research institutes where made searches to building the database of master's thesis and $\mathrm{PhD}$ dissertations with ornithological thematics

Fundação Oswaldo Cruz (FIOCRUZ), Fundação Universidade Federal do Rio Grande (FURG), Instituto Nacional de Pesquisas da Amazônia (INPA), Pontifícia Universidade Católica de Minas Gerais (PUC/MG), Pontifícia Universidade Católica do Rio Grande do Sul (PUC/RS), Universidade para o Desenvolvimento do Estado e da Região do Pantanal (UNIDERP), Universidade Católica de Brasília (UCB), Universidade Católica de Goiás (UCGO), Universidade de Brasília (UnB), Universidade de São Paulo (USP), Universidade de São Paulo/Esalq (USP/Esalq), Universidade de São Paulo/Ribeirão Preto (USP/RP), Universidade de São Paulo/São Carlos (USP/ São Carlos), Universidade do Estado do Rio de Janeiro (UERJ), Universidade do Vale do Itajaí (UNIVALI), Universidade Estadual de Campinas (UNICAMP), Universidade Estadual de Londrina (UEL), Universidade Estadual de Maringá (UEM), Universidade Estadual de Santa Cruz (UESC), Universidade Estadual de Feira de Santana (UEFS), Universidade Estadual Norte Fluminense (UENF), Universidade Estadual Paulista/Botucatu (UNESP/ Bot.), Universidade Estadual Paulista/Rio Claro (UNESP/RC), Universidade Estadual Paulista/São José do Rio Preto (UNESP/ SJRP), Universidade Federal da Bahia (UFBA), Universidade Federal da Paraíba (UFPB), Universidade Federal de Goiás (UFG), Universidade Federal de Juiz de Fora (UFJF), Universidade Federal de Lavras (UFLA), Universidade Federal de Mato Grosso (UFMT), Universidade Federal de Mato Grosso do Sul (UFMS), Universidade Federal de Minas Gerais (UFMG), Universidade Federal de Pernambuco (UFPE), Universidade Federal de Santa Maria (UFSM), Universidade Federal de Santa Catarina (UFSC), Universidade Federal de São Carlos (UFSCAR), Universidade Federal de Uberlândia (UFU), Universidade Federal de Viçosa (UFV), Universidade Federal do Acre (UFAC), Universidade Federal do Amazonas (UFAM), Universidade Federal do Pará (UFPA), Universidade Federal do Paraná (UFPR), Universidade Federal do Rio de Janeiro (UFRJ), Universidade Federal do Rio Grande do Norte (UFRN), Universidade Federal do Rio Grande do Sul (UFRGS), Universidade Federal Fluminense (UFF), Universidade Federal Rural da Amazônia (UFRA), Universidade Federal Rural de Pernambuco (UFRPE), Universidade Federal Rural do Rio de Janeiro (UFRRJ), Universidade Luterana do Brasil (ULBRA), Universidade Vale dos Sinos (UNISINOS). 